\title{
GENERALIZED BOUNDS FOR SINE AND COSINE FUNCTIONS
}

\author{
YOGESH J. BAGUL AND CHRISTOPHE CHESNEAU
}

\begin{abstract}
In this paper, we propose several new lower and upper bounds for the functions $\sin x / x$ and $\cos x$. In particular, we refine by generalizing some known inequalities involving these functions. To attain this aim, monotonicity rules and ratio of consecutive even indexed Bernoulli numbers play an important role.
\end{abstract}

\section{Introduction}

Since the last two decades, there has been a growing interest in the field of inequalities involving trigonometric functions (see $[4,6,7,10,13,15,16,18,21]$, and references therein). In this connection, R. Klén et al. [13] proved the following inequalities:

$$
1-\frac{x^{2}}{6}<\frac{\sin x}{x}<1-\frac{2 x^{2}}{3 \pi^{2}} ; 0 \in(0, \pi / 2) .
$$

These inequalities were sharpened in [5] as follows:

$$
1-\frac{x^{2}}{6}<\frac{\sin x}{x}<1-\frac{4 x^{2}}{3 \pi^{2}} ; 0 \in(0, \pi / 2) .
$$

In the same paper [5], the inequalities

$$
1-\frac{x^{2}}{2}<\cos x<1-\frac{4 x^{2}}{\pi^{2}} ; \quad x \in(0, \pi / 2)
$$

and

$$
\frac{2-x^{2}}{2}<\cos x<\frac{2}{2+x^{2}} ; \quad x \in(0, \pi / 2)
$$

were proved by Y. J. Bagul and S. K. Panchal. Another interesting inequality [3] is stated as

$$
\frac{\pi^{2}}{\pi^{2}+2(\pi-2) x^{2}}<\frac{\sin x}{x}<\frac{6}{6+x^{2}} ; \quad x \in(0, \pi / 2) .
$$

2010 Mathematics Subject Classification. 26D05, 26D07, 26D20, 33B10.

Key words and phrases. Generalized bounds, l'Hôpital's tule of monotonicity, Bernoulli numbers, trigonometric functions. 
For refinements of lower bounds, C. Chesneau and Y. J. Bagul [9] established that

$$
\left(1-\frac{x^{2}}{\pi^{2}}\right)^{\pi^{2} / 6}<\frac{\sin x}{x} ; x \in(0, \pi)
$$

and

$$
\left(1-\frac{4 x^{2}}{\pi^{2}}\right)^{\pi^{2} / 8}<\cos x ; x \in(0, \pi / 2) .
$$

Inequalities having resemblance with the above inequalities (i.e., close resemblance with (1.6) and (1.7)) can also be found in [21]. Motivated by these inequalities, and the challenge to sharpen them as well, we aim to further refine and generalize these inequalities by using new mathematical approaches.

The rest of the paper is planned as follows. Section 2 presents some useful preliminaries and lemmas. The main results are provided in Section 3. Section 4 is devoted to applications of these results.

\section{Preliminaries AND Lemmas}

First of all, we need to remind ourselves the following power series expansions $[12,1.3 .1 .4]$ :

$$
\frac{\tan x}{x}=\sum_{n=1}^{\infty} \frac{2^{2 n}\left(2^{2 n}-1\right)}{(2 n) !}\left|B_{2 n}\right| x^{2 n-2} ;|x|<\frac{\pi}{2}
$$

and

$$
x \cot x=1-\sum_{n=1}^{\infty} \frac{2^{2 n}}{(2 n) !}\left|B_{2 n}\right| x^{2 n} ;|x|<\pi .
$$

Similarly,

$$
\left(\frac{x}{\sin x}\right)^{2}=1+\sum_{n=1}^{\infty} \frac{2^{2 n}}{(2 n) !}(2 n-1)\left|B_{2 n}\right| x^{2 n} ;|x|<\pi
$$

can be found in [7, p. 128] or can be easily obtained from (2.2). Here, $B_{2 n}$ are even indexed Bernoulli numbers. With these series expansions, the following four lemmas allow us to establish our main results.

Lemma 1. ([2, p. 10]) Let $p, q:[a, b] \rightarrow \mathbb{R}$ be continuous functions. Moreover, let $p, q$ be differentiable functions on $(a, b)$, with $q^{\prime}(x) \neq 0$, on $(a, b)$. Now, set

$$
r_{1}(x)=\frac{p(x)-p(a)}{q(x)-q(a)}, r_{2}(x)=\frac{p(x)-p(b)}{q(x)-q(b)}, x \in(a, b)
$$

Then, 
(i) $r_{1}(\cdot)$ and $r_{2}(\cdot)$ are increasing(strictly increasing) on $(a, b)$ if $p^{\prime}(\cdot) / q^{\prime}(\cdot)$ is increasing(strictly increasing) on $(a, b)$.

(ii) $r_{1}(\cdot)$ and $r_{2}(\cdot)$ are decreasing $\left(\right.$ strictly decreasing) on $(a, b)$ if $p^{\prime}(\cdot) / q^{\prime}(\cdot)$ is decreasing(strictly decreasing) on $(a, b)$.

Lemma 1 is known in the literature as a monotone form of l'Hôpital's rule (see also [14]). Lemma 2 below can be found in $[1,11]$.

Lemma 2. ( $[1,11])$ Let $A(x)=\sum_{n=0}^{\infty} a_{n} x^{n}$ and $B(x)=\sum_{n=0}^{\infty} b_{n} x^{n}$ be convergent for $|x|<T$, where $a_{n}$ and $b_{n}$ are real numbers for $n=0,1,2, \cdots$ such that $b_{n}>0$., and $T>0$ is a fixed constant. If the sequence $a_{n} / b_{n}$ is strictly increasing(or decreasing), then the function $A(x) / B(x)$ is also strictly increasing(or decreasing) on $(0, T)$.

The result below is about lower and upper bounds for a ratio involving absolute Bernoulli numbers. It is established in [17].

Lemma 3. ([17]) For $k \in \mathbb{N}$, the Bernoulli numbers satisfy

$$
\frac{\left(2^{2 k-1}-1\right)}{\left(2^{2 k+1}-1\right)} \frac{(2 k+1)(2 k+2)}{\pi^{2}}<\frac{\left|B_{2 k+2}\right|}{\left|B_{2 k}\right|}<\frac{\left(2^{2 k}-1\right)}{\left(2^{2 k+2}-1\right)} \frac{(2 k+1)(2 k+2)}{\pi^{2}} .
$$

The inequality in the following lemma is known in the literature as Wilker's inequality and it is proved in [20].

Lemma 4. [20] If $x \in(0, \pi / 2)$, then

$$
\frac{\tan x}{x}+\left(\frac{\sin x}{x}\right)^{2}>2
$$

\section{MAin ReSUlts}

We will now state and prove the main results of the paper.

Theorem 1. Let the function $f_{u}(x)=\frac{\ln (\sin x / x)}{\ln \left[\left(\pi^{2}-u x^{2}\right) / \pi^{2}\right]}$ be defined on $(0, \pi / 2)$ and $u \neq 0$. Then,

1. $f_{u}(x)$ is strictly decreasing on $(0, \pi / 2)$ if $4\left(1-\frac{8}{\pi^{2}}\right) \leqslant u \leqslant 4$,

2. $f_{u}(x)$ is strictly decreasing on $(0, \pi / 2)$ if $u<0$,

3. $f_{u}(x)$ is strictly increasing on $(0, \pi / 2)$ if $0<u \leqslant \frac{\pi^{2}}{15}$.

Proof. First of all, note that $\ln \left(\frac{\pi^{2}-u x^{2}}{\pi^{2}}\right)$ is defined in $(0, \pi / 2)$ provided that $u \leqslant 4$. Now, consider

$$
f_{u}(x)=\frac{\ln \left(\frac{\sin x}{x}\right)}{\ln \left(\frac{\pi^{2}-u x^{2}}{\pi^{2}}\right)}=\frac{f_{1}(x)}{\left(f_{2}\right)_{u}(x)},
$$


where $f_{1}(x)=\ln \left(\frac{\sin x}{x}\right)$ and $\left(f_{2}\right)_{u}(x)=\ln \left(\frac{\pi^{2}-u x^{2}}{\pi^{2}}\right)$ satisfying $f_{1}\left(0^{+}\right)=0=$ $\left(f_{2}\right)_{u}\left(0^{+}\right)$. Differentiation gives

$$
\begin{aligned}
\frac{f_{1}^{\prime}(x)}{\left(f_{2}\right)_{u}^{\prime}(x)}=\frac{1}{2 u}\left(\pi^{2}-u x^{2}\right)\left(\frac{\sin x-x \cos x}{x^{2} \sin x}\right) & =\frac{1}{2 u}\left(\frac{\pi^{2}}{x^{2}}-\frac{\pi^{2} \cot x}{x}-u+u x \cot x\right) \\
& =\frac{1}{2 u}\left(f_{3}\right)_{u}(x) .
\end{aligned}
$$

Clearly, $\left(f_{3}\right)_{u}(x)$ is decreasing if and only if $\left(f_{3}\right)_{u}^{\prime}(x)<0$. Consequently, by using Lemma 1, we can conclude that $f_{u}(x)$ will be decreasing if $\left(f_{3}\right)_{u}^{\prime}(x)<0$. This implies that

or

$$
-2 \pi^{2}+\pi^{2}\left(\frac{x}{\sin x}\right)^{2}+\pi^{2} \frac{x}{\tan x}+u x^{2} \frac{x}{\tan x}-u x^{2}\left(\frac{x}{\sin x}\right)^{2}<0
$$

$$
\pi^{2}\left[\left(\frac{x}{\sin x}\right)^{2}+x \cot x-2\right]<u x^{2}\left[\left(\frac{x}{\sin x}\right)^{2}-x \cot x\right]
$$

This inequality is equivalent to

$$
\frac{\pi^{2}\left[\left(\frac{x}{\sin x}\right)^{2}+x \cot x-2\right]}{x^{2}\left[\left(\frac{x}{\sin x}\right)^{2}-x \cot x\right]}<u,
$$

since the relation $\frac{x}{\tan x}<1<\left(\frac{x}{\sin x}\right)^{2}$ is well known. Set $f_{4}(x)=\frac{\left(\frac{x}{\sin x}\right)^{2}+x \cot x-2}{x^{2}\left[\left(\frac{x}{\sin x}\right)^{2}-x \cot x\right]}$ so that $f_{4}(x)<\frac{u}{\pi^{2}}$. Owing to $(2.2)$ and $(2.3)$, we have

$$
\begin{aligned}
f_{4}(x) & =\frac{\sum_{n=1}^{\infty} \frac{2^{2 n}}{(2 n) !}(2 n-2)\left|B_{2 n}\right| x^{2 n}}{\sum_{n=1}^{\infty} \frac{2^{2 n}}{(2 n) !} 2 n\left|B_{2 n}\right| x^{2 n+2}} \\
& =\frac{\sum_{n=2}^{\infty} \frac{2^{2 n}}{(2 n) !}(2 n-2)\left|B_{2 n}\right| x^{2 n}}{\sum_{n=2}^{\infty} \frac{2^{2 n-2}}{(2 n-2) !}(2 n-2)\left|B_{2 n-2}\right| x^{2 n}} \\
& =\frac{\sum_{n=2}^{\infty} a_{n} x^{2 n}}{\sum_{n=2}^{\infty} b_{n} x^{2 n}}
\end{aligned}
$$

where $a_{n}=\frac{2^{2 n}}{(2 n) !}(2 n-2)\left|B_{2 n}\right|$ and $b_{n}=\frac{2^{2 n-2}}{(2 n-2) !}(2 n-2)\left|B_{2 n-2}\right|>0$.

Therefore, with $c_{n}=\frac{a_{n}}{b_{n}}$, we get

$$
c_{n}=\frac{a_{n}}{b_{n}}=\frac{4\left|B_{2 n}\right|}{2 n(2 n-1)\left|B_{2 n-2}\right|}
$$

and

$$
\frac{c_{n+1}}{c_{n}}=\frac{2 n(2 n-1)}{(2 n+1)(2 n+2)} \frac{\left|B_{2 n-2}\right|}{\left|B_{2 n}\right|} \frac{\left|B_{2 n+2}\right|}{\left|B_{2 n}\right|} .
$$


Lemma 3 yields

$$
\frac{\left|B_{2 n-2}\right|}{\left|B_{2 n}\right|}>\frac{\left(2^{2 n-1}-1\right)}{\left(2^{2 n-3}-1\right)} \frac{\pi^{2}}{2 n(2 n-1)}, \frac{\left|B_{2 n+2}\right|}{\left|B_{2 n}\right|}>\frac{\left(2^{2 n-1}-1\right)}{\left(2^{2 n+1}-1\right)} \frac{(2 n+1)(2 n+2)}{\pi^{2}} .
$$

This gives

$$
\frac{c_{n+1}}{c_{n}}>\frac{\left(2^{2 n-1}-1\right)^{2}}{\left(2^{2 n-3}-1\right)\left(2^{2 n+1}-1\right)} .
$$

Now, we claim that $\frac{c_{n+1}}{c_{n}}>1$. Indeed, if $\frac{c_{n+1}}{c_{n}} \leqslant 1$ for $n \geqslant 2$, then $\left(2^{2 n-1}-1\right)^{2} \leqslant$ $\left(2^{2 n-3}-1\right)\left(2^{2 n+1}-1\right)$ or $2^{2 n} \geqslant 2^{2 n-3}+2^{2 n+1} \geqslant 2^{2 n+1}$, which is absurd. Thus the sequence $\left\{\frac{a_{n}}{b_{n}}\right\}$ is strictly increasing. By Lemma 2, $f_{4}(x)$ is also strictly increasing on $(0, \pi / 2)$. Hence, $\sup \left\{f_{4}(x): x \in(0, \pi / 2)\right\}=\lim _{x \rightarrow \pi / 2} f_{4}(x)=$ $\frac{4\left(\pi^{2}-8\right)}{\pi^{4}} \leqslant \frac{u}{\pi^{2}}$. Thus $\left(f_{3}\right)_{u}(x)$ and $f_{u}(x)$ are strictly decreasing on $(0, \pi / 2)$ if $4\left(1-\frac{8}{\pi^{2}}\right) \leqslant u \leqslant 4$. For second and third statements, we follow similar arguments and conclude that $\left(f_{3}\right)_{u}(x)$ is strictly increasing on $(0, \pi / 2)$ if $\frac{u}{\pi^{2}} \leqslant$ $\inf \left\{f_{4}(x): x \in(0, \pi / 4)\right\}=\lim _{x \rightarrow 0^{+}} f_{4}(x)=\frac{1}{15}$, i.e., $u \leqslant \frac{\pi^{2}}{15}$. This ends the proof of Theorem 1 .

The statement of Theorem 1 can be extended on $(0, \pi)$ as presented below.

Theorem 2. Let the function $f_{u}(x)$ be defined as in Theorem 1 on $(0, \pi)$ and $u \neq 0$. Then,

1. $f_{u}(x)$ is strictly decreasing on $(0, \pi)$ if $u=1$,

2. $f_{u}(x)$ is strictly decreasing on $(0, \pi)$ if $u<0$,

3. $f_{u}(x)$ is strictly increasing on $(0, \pi)$ if $0<u \leqslant \frac{\pi^{2}}{15}$.

Proof. We omit the proof since it is very similar to the proof of Theorem 1.

We in turn have a generalization of first parts of Theorems 1 and 2 as follows.

Theorem 3. If $u \geqslant 1$, then the function $f_{u}(x)$ defined in Theorem 1, is decreasing on $(0, \pi / \sqrt{u})$. In particular, with this fixed value of $u$, if $x \in(0, \mu]$ where $\mu \in(0, \pi / \sqrt{u})$, then the best possible constants $\alpha$ and $\beta$ such that

$$
\left(1-\frac{u x^{2}}{\pi^{2}}\right)^{\alpha}<\frac{\sin x}{x}<\left(1-\frac{u x^{2}}{\pi^{2}}\right)^{\beta}
$$

are $\frac{\pi^{2}}{6 u}$ and $\frac{\ln (\sin \mu / \mu)}{\ln \left(1-u \mu^{2} / \pi^{2}\right)}$ respectively.

Proof. The proof is similar to that of Theorem 1. Let us just mention that

$$
\lim _{x \rightarrow 0^{+}} f_{u}(x)>f_{u}(x)>\lim _{x \rightarrow \mu^{-}} f_{u}(x)
$$

and the $\operatorname{limits}_{\lim _{x \rightarrow 0^{+}}} f_{u}(x)=\frac{\pi^{2}}{6 u}$ and $\lim _{x \rightarrow \mu^{-}} f_{u}(x)=\frac{\ln (\sin \mu / \mu)}{\ln \left(1-u \mu^{2} / \pi^{2}\right)}$ give the inequalities (3.1). 
Corollary 1. If $x \in(0, \pi / 2)$ and $4\left(1-\frac{8}{\pi^{2}}\right) \leqslant u \leqslant 4$, then the best possible constants $\alpha$ and $\beta$ such that

$$
\left(1-\frac{u x^{2}}{\pi^{2}}\right)^{\alpha}<\frac{\sin x}{x}<\left(1-\frac{u x^{2}}{\pi^{2}}\right)^{\beta}
$$

are $\frac{\pi^{2}}{6 u}$ and $\frac{\ln (2 / \pi)}{\ln (1-u / 4)}$, respectively.

Proof. As $4\left(1-\frac{8}{\pi^{2}}\right) \leqslant u \leqslant 4$, by Theorem $1, f_{u}(x)$ is strictly decreasing on $(0, \pi / 2)$. So, noticing that

$$
\lim _{x \rightarrow 0^{+}} f_{u}(x)=\frac{\pi^{2}}{6 u}>f_{u}(x)>\lim _{x \rightarrow \pi / 2^{-}} f_{u}(x)=\frac{\ln (2 / \pi)}{\ln (1-u / 4)},
$$

the proof is complete.

Figure 1 illustrates Corollary 1 by plotting the following two dimensional functions:

$$
A(x, u)=\frac{\sin x}{x}-\left(1-\frac{u x^{2}}{\pi^{2}}\right)^{\alpha}, \quad B(x, u)=\left(1-\frac{u x^{2}}{\pi^{2}}\right)^{\beta}-\frac{\sin x}{x}
$$

for $x \in(0, \pi / 2)$ and $4\left(1-\frac{8}{\pi^{2}}\right) \leqslant u \leqslant 4$.

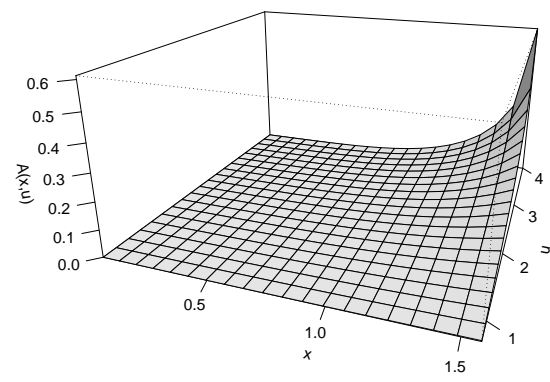

(a)

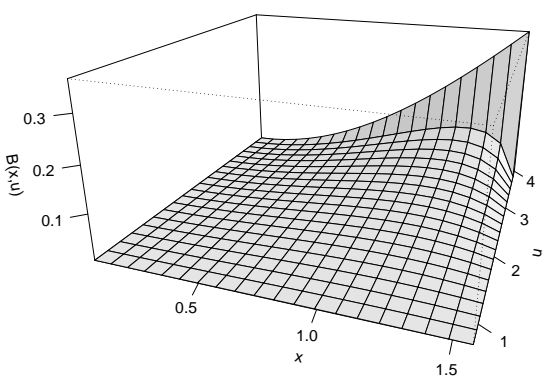

(b)

Figure 1. Plots of (a) $A(x, u)$ and (b) $B(x, u)$ for $x \in(0, \pi / 2)$ and $4\left(1-\frac{8}{\pi^{2}}\right) \leqslant u \leqslant 4$.

From Figure 1, we visually observe the findings; we have $A(x, u)>0$ and $B(x, u)>0$ for the considered values of $x$ and $u$.

On the similar line we can have following corollaries. 
Corollary 2. If $x \in(0, \mu]$ where $\mu \in(0, \pi)$ and $u=1$, then the best possible constants $\alpha$ and $\beta$ such that

$$
\left(1-\frac{u x^{2}}{\pi^{2}}\right)^{\alpha}<\frac{\sin x}{x}<\left(1-\frac{u x^{2}}{\pi^{2}}\right)^{\beta}
$$

are $\frac{\pi^{2}}{6 u}$ and $\frac{\ln (\sin \mu / \mu)}{\ln \left[\left(\pi^{2}-u \mu^{2}\right) / \pi^{2}\right]}$, respectively.

Corollary 3. If $u \in(-\infty, 0) \cup\left(0, \frac{\pi^{2}}{15}\right]$, then the best possible constants $\alpha$ and $\beta$ such that

$$
\left(1-\frac{u x^{2}}{\pi^{2}}\right)^{\beta}<\frac{\sin x}{x}<\left(1-\frac{u x^{2}}{\pi^{2}}\right)^{\alpha}
$$

are:

1. $\frac{\pi^{2}}{6 u}$ and $\frac{\ln (2 / \pi)}{\ln (1-u / 4)}$, respectively, if $x \in(0, \pi / 2)$.

2. $\frac{\pi^{2}}{6 u}$ and $\frac{\ln (\sin \mu / \mu)}{\ln \left[\left(\pi^{2}-u \mu^{2}\right) / \pi^{2}\right]}$, respectively, if $x \in(0, \mu]$ and $\mu \in(0, \pi)$.

The proof of Theorem 3 necessarily uses proof of Theorem 1 and it should be remembered that its proof can be given independently. To get the idea of independent proof of Theorem 3, we state and prove similar statement for cosine function.

Theorem 4. If $u \geqslant 4$, then the function $g_{u}(x)=\frac{\ln (\cos x)}{\ln \left(1-u x^{2} / \pi^{2}\right)}$ is decreasing on $(0, \pi / \sqrt{u})$. In particular, with this fixed value of $u$, if $x \in(0, \mu]$ where $\mu \in(0, \pi / \sqrt{u})$, then the best possible constants $\eta$ and $\xi$ such that

$$
\left(1-\frac{u x^{2}}{\pi^{2}}\right)^{\eta}<\cos x<\left(1-\frac{u x^{2}}{\pi^{2}}\right)^{\xi}
$$

are $\frac{\pi^{2}}{2 u}$ and $\frac{\ln (\cos \mu)}{\ln \left(1-u \mu^{2} / \pi^{2}\right)}$, respectively.

Proof. Let us consider

$$
g_{u}(x)=\frac{\ln (\cos x)}{\ln \left(1-\frac{u x^{2}}{\pi^{2}}\right)}=\frac{g_{1}(x)}{\left(g_{2}\right)_{u}(x)},
$$

where $g_{1}(x)=\ln (\cos x)$ and $\left(g_{2}\right)_{u}(x)=\ln \left(1-\frac{u x^{2}}{\pi^{2}}\right)$ satisfying $g_{1}(0)=0=$ $\left(g_{2}\right)_{u}(0)$. Upon differentiation, we get

$$
\frac{g_{1}^{\prime}(x)}{\left(g_{2}\right)_{u}^{\prime}(x)}=\frac{1}{2 u}\left(\pi^{2}-u x^{2}\right) \frac{\tan x}{x}=\frac{1}{2 u}\left(g_{3}\right)_{u}(x)
$$


where $\left(g_{3}\right)_{u}(x)=\left(\pi^{2}-u x^{2}\right) \frac{\tan x}{x}=\left(\pi^{2}-u x^{2}\right) \sum_{n=1}^{\infty} \frac{2^{2 n}\left(2^{2 n}-1\right)}{(2 n) !}\left|B_{2 n}\right| x^{2 n-2}$ by (2.1). After several simplifications, we arrive at

$$
\begin{aligned}
\left(g_{3}\right)_{u}(x) & =\sum_{n=1}^{\infty} \frac{\pi^{2} 2^{2 n}\left(2^{2 n}-1\right)}{(2 n) !}\left|B_{2 n}\right| x^{2 n-2}-u \sum_{n=1}^{\infty} \frac{2^{2 n}\left(2^{2 n}-1\right)}{(2 n) !}\left|B_{2 n}\right| x^{2 n} \\
& =\sum_{n=0}^{\infty} \frac{\pi^{2} 2^{2 n+2}\left(2^{2 n+2}-1\right)}{(2 n+2) !}\left|B_{2 n+2}\right| x^{2 n}-\sum_{n=1}^{\infty} \frac{2^{2 n} u\left(2^{2 n}-1\right)}{(2 n) !}\left|B_{2 n}\right| x^{2 n} \\
& =\pi^{2}+\sum_{n=1}^{\infty} a_{n} x^{2 n} .
\end{aligned}
$$

It has derivative

$$
\left(g_{3}\right)_{u}^{\prime}(x)=\sum_{n=1}^{\infty} 2 n a_{n} x^{2 n-1},
$$

where $a_{n}=\frac{\pi^{2} 2^{2 n+2}\left(2^{2 n+2}-1\right)}{(2 n+2) !}\left|B_{2 n+2}\right|-\frac{2^{2 n} u\left(2^{2 n}-1\right)}{(2 n) !}\left|B_{2 n}\right|$. From the right inequality of Lemma 3 , we obtain

$$
\pi^{2}\left(2^{2 n+2}-1\right)\left|B_{2 n+2}\right|<\left(2^{2 n}-1\right)(2 n+1)(2 n+2)\left|B_{2 n}\right| .
$$

This yields, with $a \geqslant 4$,

$$
\begin{aligned}
\frac{\pi^{2} 2^{2 n+2}\left(2^{2 n+2}-1\right)}{(2 n+2) !}\left|B_{2 n+2}\right| & <\frac{2^{2 n} 2^{2}\left(2^{2 n}-1\right)}{(2 n) !}\left|B_{2 n}\right| \\
& \leqslant \frac{2^{2 n} u\left(2^{2 n}-1\right)}{(2 n) !}\left|B_{2 n}\right| .
\end{aligned}
$$

Thus, $a_{n} \leqslant 0 ; \forall n \in \mathbb{N}$ and consequently, $\left(g_{3}\right)_{u}(x)$, and hence $g_{u}(x)$, are decreasing in $(0, \pi / \sqrt{u})$. Lastly the relation $\lim _{x \rightarrow 0^{+}} g_{u}(x)=\frac{\pi^{2}}{2 u}>g_{u}(x)>$ $\lim _{x \rightarrow \mu^{-}} g_{u}(x)=\frac{\ln (\cos \mu)}{\ln \left(1-u \mu^{2} / \pi^{2}\right)}$ proves the inequalities (3.5).

Before giving an enhanced statement similar to the one of Theorem 1 for $g_{u}(x)$, we first provide an alternative proof to the result of Sándor-Bhayo [19, Theorem 1].

Theorem 5. ( [19, Theorem 1]) The function $P(x)=\frac{2 x-\sin 2 x}{x^{2}(2 x+\sin 2 x)}$ is strictly increasing on $(0, \pi / 2)$. In particular, the inequalities

$$
\frac{\pi^{2}-x^{2}}{\pi^{2}+x^{2}}<\frac{\sin x}{x}<\frac{12-x^{2}}{12+x^{2}}
$$

are true in $(0, \pi]$.

Proof. Let

$$
P(x)=\frac{2 x-\sin 2 x}{x^{2}(2 x+\sin 2 x)}=\frac{2 x-\sin 2 x}{2 x^{3}+x^{2} \sin 2 x} .
$$


Differentiation gives

$$
\begin{aligned}
\left(2 x^{3}+x^{2} \sin 2 x\right)^{2} P^{\prime}(x) & =-8 x^{3}-8 x^{3} \cos 2 x+4 x^{2} \sin 2 x+2 x \sin ^{2} 2 x \\
& =8 x^{2} \cos x(\sin x-x \cos x)+8 x \cos ^{2} x\left(\sin ^{2} x-x^{2}\right) \\
& =8 x \cos x Q(x),
\end{aligned}
$$

where $Q(x)=x \sin x+\sin ^{2} x \cos x-2 x^{2} \cos x>0$ by Lemma 4 . The limits at the extremities give the inequalities (3.6).

We are now in the position to state and prove an enhanced statement of Theorem 4.

Theorem 6. Let the function $g_{u}(x)$ be defined as in Theorem 4 on $(0, \pi / 2)$ and $u \neq 0$. Then

1. $g_{u}(x)$ is strictly decreasing on $(0, \pi / 2)$ if $u=4$,

2. $g_{u}(x)$ is strictly decreasing on $(0, \pi / 2)$ if $u<0$,

3. $g_{u}(x)$ is strictly increasing on $(0, \pi / 2)$ if $0<u \leqslant \frac{\pi^{2}}{3}$.

Proof. As in the proof of Theorem 4, we have $u \leqslant 4$ and

$$
\frac{g_{1}^{\prime}(x)}{\left(g_{2}\right)_{u}^{\prime}(x)}=\frac{1}{2 u}\left(\pi^{2}-u x^{2}\right) \frac{\tan x}{x}=\frac{1}{2 u}\left(g_{3}\right)_{u}(x)
$$

where $\left(g_{3}\right)_{u}(x)=\left(\pi^{2}-u x^{2}\right) \frac{\tan x}{x}$. It has the following derivative

$$
\begin{aligned}
\left(g_{3}\right)_{u}^{\prime}(x) & =\frac{1}{x^{2}}\left(x\left[\left(\pi^{2}-u x^{2}\right) \sec ^{2} x-2 u x \tan x\right]-\left(\pi^{2}-u x^{2}\right) \tan x\right) \\
& =\pi^{2}\left(x \sec ^{2} x-\tan x\right)-u x^{2}\left(\tan x+x \sec ^{2} x\right) .
\end{aligned}
$$

Clearly, $\left(g_{3}\right)_{u}(x)$ is decreasing if and only if $\left(g_{3}\right)_{u}^{\prime}(x)<0$. Consequently, by using Lemma 1, we can conclude that $g_{u}(x)$ will be decreasing if $\left(g_{3}\right)_{u}^{\prime}(x)<0$ which implies that

$$
u x^{2}\left(\tan x+x \sec ^{2} x\right)>\pi^{2}\left(x \sec ^{2} x-\tan x\right),
$$

i.e.,

$$
\frac{u}{\pi^{2}}>\frac{x \sec ^{2} x-\tan x}{x^{2}\left(x \sec ^{2} x+\tan x\right)}=\frac{2 x-\sin 2 x}{x^{2}(2 x+\sin 2 x)}=P(x) .
$$

By virtue of Theorem 5, we can write $\frac{u}{\pi^{2}} \geqslant \sup \{P(x): x \in(0, \pi / 2)\}=$ $\lim _{x \rightarrow \pi / 2^{-}} P(x)=\frac{4}{\pi^{2}}$, i.e., $u \geqslant 4$. Thus, $\left(g_{3}\right)_{u}(x)$ is strictly decreasing if $u \leqslant 4$ and $u \geqslant 4$ i.e., $u=4$. Hence $g_{u}(x)$ is strictly decreasing on $(0, \pi / 2)$ if $u=4$. For second and third statements, we follow the similar arguments and conclude that $\left(g_{3}\right)_{u}(x)$ is strictly increasing on $(0, \pi / 2)$ if $\frac{u}{\pi^{2}} \leqslant \inf \{P(x): x \in(0, \pi / 2)\}=$ $\lim _{x \rightarrow 0^{+}} P(x)=\frac{1}{3}$, i.e., $u \leqslant \frac{\pi^{2}}{3}$. This completes the proof.

As a consequence of Theorem 6, we have following direct corollaries. 
Corollary 4. If $x \in(0, \lambda]$ where $\lambda \in(0, \pi / 2)$ and $u=4$, then the best possible constants $\eta$ and $\zeta$ such that

$$
\left(1-\frac{u x^{2}}{\pi^{2}}\right)^{\eta}<\cos x<\left(1-\frac{u x^{2}}{\pi^{2}}\right)^{\zeta}
$$

are $\frac{\pi^{2}}{2 u}$ and $\frac{\ln (\cos \lambda)}{\ln \left[\left(\pi^{2}-u \lambda^{2}\right) / \pi^{2}\right]}$, respectively.

Proof. By the first statement of Theorem 6 and the use of the $\operatorname{limits}_{\lim } \lim _{x \rightarrow 0^{+}} g_{u}(x)=$ $\frac{\pi^{2}}{2 u}$ and $\lim _{x \rightarrow \lambda^{-}} g_{u}(x)=\frac{\ln (\sin \lambda)}{\ln \left[\left(\pi^{2}-u \lambda^{2}\right) / \pi^{2}\right]}$, we get the required result.

Corollary 5. If $u \in(-\infty, 0) \cup\left(0, \frac{\pi^{2}}{3}\right]$ and $x \in(0, \lambda]$ where $\lambda \in(0, \pi / 2)$, then the best possible constants $\eta$ and $\zeta$ such that

$$
\left(1-\frac{u x^{2}}{\pi^{2}}\right)^{\zeta}<\cos x<\left(1-\frac{u x^{2}}{\pi^{2}}\right)^{\eta}
$$

are $\frac{\pi^{2}}{2 u}$ and $\frac{\ln (\cos \lambda)}{\ln \left[\left(\pi^{2}-u \lambda^{2}\right) / \pi^{2}\right]}$, respectively.

Figure 2 illustrates visually Corollary 5 by considering the two dimensional functions:

$$
C(x, u)=\left(1-\frac{u x^{2}}{\pi^{2}}\right)^{\eta}-\cos x, \quad D(x, u)=\cos x-\left(1-\frac{u x^{2}}{\pi^{2}}\right)^{\zeta}
$$

for $u \in\left(0, \frac{\pi^{2}}{3}\right]$ and $x \in(0, \lambda]$ where $\lambda=\pi / 3$. 


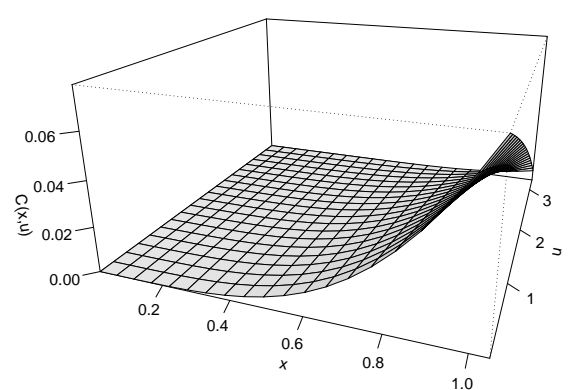

(a)

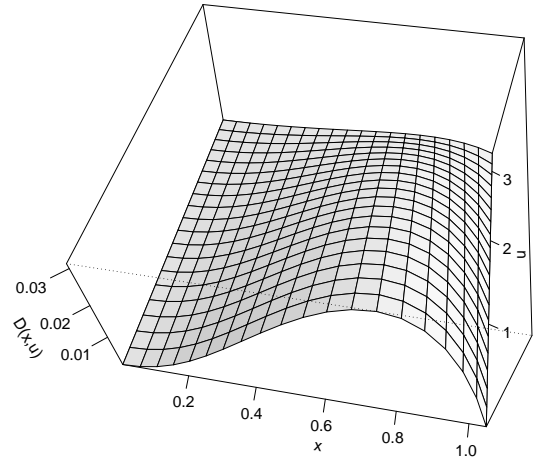

(b)

Figure 2. Plots of (a) $C(x, u)$ and (b) $D(x, u)$ for $u \in\left(0, \frac{\pi^{2}}{3}\right]$ and $x \in(0, \lambda]$ where $\lambda=\pi / 3$.

From Figure 2, as proved analytically, we see that $C(x, u)>0$ and $D(x, u)>$ 0 for the considered values of $x$ and $u$.

\section{Applications}

Inequalities (1.6) and (1.7) are particular cases of Corollary 2 (or Theorem 3) and Corollary 4 (or Theorem 4), respectively, with their extensions to the right also. Now, putting $u=\pi^{2} / 6$ and $u=4 / 3$ in (3.2), we get, respectively, the following inequalities:

$$
1-\frac{x^{2}}{6}<\frac{\sin x}{x}<\left(1-\frac{x^{2}}{6}\right)^{\beta_{1}} ; x \in(0, \pi / 2),
$$

and

$$
\left(1-\frac{4 x^{2}}{3 \pi^{2}}\right)^{\pi^{2} / 8}<\frac{\sin x}{x}<\left(1-\frac{4 x^{2}}{3 \pi^{2}}\right)^{\beta_{2}} ; x \in(0, \pi / 2)
$$

with $\beta_{1}=\frac{\ln (2 / \pi)}{\ln \left(1-\pi^{2} / 24\right)} \approx 0.85248$ and $\beta_{2}=\frac{\ln (2 / \pi)}{\ln (2 / 3)} \approx 1.113739$.

Obviously, the inequalities (4.1) and (4.2) refine those in (1.1) and (1.2). By fixing $u=1$ in Corollary 1, we get refinement of both (4.1) and (4.2). The 
refined inequalities can be formulated as follows:

$$
\left(1-\frac{x^{2}}{\pi^{2}}\right)^{\pi^{2} / 6}<\frac{\sin x}{x}<\left(1-\frac{x^{2}}{\pi^{2}}\right)^{\beta_{3}} ; x \in(0, \pi / 2)
$$

with $\beta_{3}=\frac{\ln (2 / \pi)}{\ln (3 / 4)} \approx 1.569728$.

Similarly after fixing $u=4 / 5$ in Corollary 1 , we get

$$
\left(1-\frac{4 x^{2}}{5 \pi^{2}}\right)^{5 \pi^{2} / 24}<\frac{\sin x}{x}<\left(1-\frac{4 x^{2}}{5 \pi^{2}}\right)^{\beta_{4}} ; x \in(0, \pi / 2),
$$

where $\beta_{4}=\frac{\ln (2 / \pi}{\ln (4 / 5)} \approx 2.023732$.

We found sharpest double inequality among all the inequalities in (3.2) by fixing least value of $u$ in Corollary 1 as follows:

$$
\left[1-4\left(1-\frac{8}{\pi^{2}}\right) \frac{x^{2}}{\pi^{2}}\right]^{\frac{\pi^{2}}{24\left(1-8 / \pi^{2}\right)}}<\frac{\sin x}{x}<\left[1-4\left(1-\frac{8}{\pi^{2}}\right) \frac{x^{2}}{\pi^{2}}\right]^{\beta_{5}} ; x \in(0, \pi / 2)
$$

where $\beta_{5}=\frac{\ln (2 / \pi)}{\ln \left(8 / \pi^{2}\right)} \approx 2.150207$.

Again, from Corollary 3, we obtain the following better inequalities by putting respectively $u=\frac{\pi^{2}}{15}$ and $u=\frac{4}{7}$, obtaining

$$
\left(1-\frac{x^{2}}{15}\right)^{\beta_{6}}<\frac{\sin x}{x}<\left(1-\frac{x^{2}}{15}\right)^{5 / 2} ; x \in(0, \pi / 2)
$$

and

$$
\left(1-\frac{4 x^{2}}{7 \pi^{2}}\right)^{\beta_{7}}<\frac{\sin x}{x}<\left(1-\frac{4 x^{2}}{7 \pi^{2}}\right)^{7 \pi^{2} / 24} ; x \in(0, \pi / 2)
$$

where $\beta_{6}=\frac{\ln (2 / \pi)}{\ln \left(1-\pi^{2} / 60\right)} \approx 2.512743$, and $\beta_{7}=\frac{\ln (2 / \pi)}{\ln (6 / 7)} \approx 2.929488$,

Remark 1. There is no strict comparison among the inequalities in (4.5), (4.6) and (4.7).

A refinement of (1.5) is obtained by putting $u=-\frac{\pi^{2}}{6}$ in Corollary 3 as follows:

$$
\left(\frac{6}{6+x^{2}}\right)^{\beta_{8}}<\frac{\sin x}{x}<\frac{6}{6+x^{2}} ; \quad x \in(0, \pi / 2)
$$


where $\beta_{8}=-\frac{\ln (2 / \pi)}{\ln \left(1+\pi^{2} / 24\right)} \approx 1.310971$. For better refinement of inequalities of type (4.8), we can put the negative values of $u$ in Corollary 3 such that $|u|$ is least possible. For example, putting $u=-1$ in Corollary 3 we get

$$
\left(\frac{\pi^{2}}{\pi^{2}+x^{2}}\right)^{\beta_{9}}<\frac{\sin x}{x}<\left(\frac{\pi^{2}}{\pi^{2}+x^{2}}\right)^{\pi^{2} / 6} ; x \in(0, \pi / 2),
$$

where $\beta_{9}=-\frac{\ln (2 / \pi)}{5 / 4)} \approx 2.023732$ and it is still possible to get sharper and sharper inequalities involving $\frac{\sin x}{x}$.

For inequalities involving $\cos x$, we directly obtain from Corollary 5 , the following inequalities for $x \in(0, \lambda]$ :

$$
\begin{gathered}
\left(1-\frac{x^{2}}{3}\right)^{\zeta_{1}}<\cos x<\left(1-\frac{x^{2}}{3}\right)^{3 / 2} \\
\left(\frac{2}{2+x^{2}}\right)^{\zeta_{2}}<\cos x<\frac{2}{2+x^{2}}
\end{gathered}
$$

and

$$
\left(\frac{\pi^{2}}{\pi^{2}+x^{2}}\right)^{\zeta_{3}}<\cos x<\left(\frac{\pi^{2}}{\pi^{2}+x^{2}}\right)^{\pi^{2} / 2}
$$

where $\lambda \in(0, \pi / 2), \zeta_{1}=\frac{\ln (\cos \lambda)}{\ln \left(1-\lambda^{2} / 3\right)}, \quad \zeta_{2}=-\frac{\ln (\cos \lambda)}{\ln \left(1+\lambda^{2} / 2\right)}$ and $\zeta_{3}=-\frac{\ln (\cos \lambda)}{\ln \left(1+\lambda^{2} / \pi^{2}\right)}$. Note that the right inequalities of (4.10), (4.11) and (4.12) are in fact true in $(0, \pi / 2)$. The right inequality of $(4.10)$ can also be seen in [8, Lemma 2.9]. The double inequality (4.11) is sharpened version of (1.4) and (4.12) further refines (4.11).

Lastly, combining different inequalities we get inequalities of great interest. For instance, from (1.6) and (4.9) we have

$$
\left(\frac{\pi^{2}-x^{2}}{\pi^{2}}\right)^{\pi^{2} / 6}<\frac{\sin x}{x}<\left(\frac{\pi^{2}}{\pi^{2}+x^{2}}\right)^{\pi^{2} / 6} ; x \in(0, \pi) .
$$

Note here that the right inequality in $(4.13)$ holds in $(0, \pi)$ due to Theorem 1 , 3 (Corollary 2). Another one for $\cos x$ is given as

$$
\left(\frac{\pi^{2}-4 x^{2}}{\pi^{2}}\right)^{\pi^{2} / 8}<\cos x<\left(\frac{\pi^{2}}{\pi^{2}+4 x^{2}}\right)^{\pi^{2} / 8} ; x \in(0, \pi / 2),
$$

where the left inequality is (1.7) and right inequality are a consequence of Theorem 6(Corollary 5). Thus, we can establish several inequalities of type (4.13) and (4.14). 
For another interesting application, one can consider the following chain of inequalities obtained by E. Neuman [16]:

$$
\begin{aligned}
(\cos x)^{1 / 3} & <\left(\cos x \frac{\sin x}{x}\right)^{1 / 4}<\frac{\sin x}{\arctan (\sin x)}<\left(\frac{\cos x+\sin x / x}{2}\right)^{1 / 2} \\
& <\left(\frac{1+2 \cos x}{3}\right)^{1 / 2}<\left(\frac{1+\cos x}{2}\right)^{2 / 3}<\frac{\sin x}{x} ; x \in(0, \pi / 2) .
\end{aligned}
$$

To add a piece to this chain of inequalities, we first prove the two following propositions.

Proposition 1. If $x \in(0, \pi / 2)$, then we have

$$
\frac{3}{2}\left[\left(1-\frac{x^{2}}{\pi^{2}}\right)^{\pi^{2} / 3}-\left(\frac{3}{4}\right)^{\pi^{2} / 3}\right]<\cos x<\frac{1}{2}\left[3\left(1-\frac{x^{2}}{\pi^{2}}\right)^{\pi^{2} / 3}-1\right] .
$$

Proof. Set

$$
h(x)=\frac{3}{2}\left(1-\frac{x^{2}}{\pi^{2}}\right)^{\pi^{2} / 3}-\cos x .
$$

On differentiating, we get

$$
h^{\prime}(x)=\sin x-x\left(1-\frac{x^{2}}{\pi^{2}}\right)^{\frac{\pi^{2}-3}{3}}>0,
$$

since $\frac{\pi^{2}-3}{3}>\frac{\pi^{2}}{6}$ implies $\left(1-\frac{x^{2}}{\pi^{2}}\right)^{\frac{\pi^{2}-3}{3}}<\left(1-\frac{x^{2}}{\pi^{2}}\right)^{\frac{\pi^{2}}{6}}$ and $\left(1-\frac{x^{2}}{\pi^{2}}\right)^{\frac{\pi^{2}}{6}}<\frac{\sin x}{x}$ by (3.2). Therefore, $h(x)$ is strictly increasing in $(0, \pi / 2)$. Hence, we have $h(0)<h(x)<h(\pi / 2)$, i.e., $\frac{1}{2}<h(x)<\frac{3}{2}\left(\frac{3}{4}\right)^{\pi^{2} / 3}$, which proves the proposition.

Proposition 2. If $x \in(0, \pi / 2)$, then we have

$$
2\left(1-\frac{x^{2}}{\pi^{2}}\right)^{\pi^{2} / 4}-1<\cos x<2\left(1-\frac{x^{2}}{\pi^{2}}\right)^{\pi^{2} / 4}-2\left(\frac{3}{4}\right)^{\pi^{2} / 4} .
$$

Proof. Set

$$
k(x)=2\left(1-\frac{x^{2}}{\pi^{2}}\right)^{\pi^{2} / 4}-\cos x .
$$

After differentiating, we have

$$
k^{\prime}(x)=\sin x-x\left(1-\frac{x^{2}}{\pi^{2}}\right)^{\frac{\pi^{2}-4}{4}}<0,
$$


since $\frac{\pi^{2}-4}{4} \approx 1.467401<\beta_{3} \approx 1.569728$ implies that $\left(1-\frac{x^{2}}{\pi^{2}}\right)^{\beta_{3}}<\left(1-\frac{x^{2}}{\pi^{2}}\right)^{\frac{\pi^{2}-4}{4}}$ and $\frac{\sin x}{x}<\left(1-\frac{x^{2}}{\pi^{2}}\right)^{\beta_{3}}$ by (4.3). Thus, $k(x)$ is strictly decreasing in $(0, \pi / 2)$. Hence, we have $k(0)>k(x)>k(\pi / 2)$, i.e., $1>k(x)>2\left(\frac{3}{4}\right)^{\pi^{2} / 4}$. The proposition is proved.

Now, the rearrangement and combination of the right inequality of (4.8) and left inequality of (4.9) give us

$$
\left(\frac{1+2 \cos x}{3}\right)^{1 / 2}<\left(1-\frac{x^{2}}{\pi^{2}}\right)^{\pi^{2} / 6}<\left(\frac{1+\cos x}{2}\right)^{2 / 3} ; x \in(0, \pi / 2) .
$$

The chain of inequalities in (4.7) is extended as

$$
\begin{gathered}
(\cos x)^{1 / 3}<\left(\cos x \frac{\sin x}{x}\right)^{1 / 4}<\frac{\sin x}{\arctan (\sin x)}<\left(\frac{\cos x+\sin x / x}{2}\right)^{1 / 2} \\
<\left(\frac{1+2 \cos x}{3}\right)^{1 / 2}<\left(1-\frac{x^{2}}{\pi^{2}}\right)^{\pi^{2} / 6}<\left(\frac{1+\cos x}{2}\right)^{2 / 3}<\frac{\sin x}{x} ; x \in(0, \pi / 2) .
\end{gathered}
$$

\section{REFERENCES}

[1] H. Alzer, and S. L. Qiu, Monotonicity theorems and inequalities for the complete elliptic integrals, J. Comput. Appl. Math., 172, pp. 289-312, 2004.

[2] G. D. Anderson, M. K. Vamanamurthy and M. Vuorinen, Conformal Invarients, Inequalities and Quasiconformal maps, John Wiley and Sons, New York, 1997.

[3] Y. J. Bagul, On simple Jordan type inequalities, Turkish Journal of Inequalities, Vol. 3, No. 1, 2019, pp. 1-6.

[4] Y. J. Bagul and C. Chesneau, Some new simple inequalities involving exponential, trigonometric and hyperbolic functions, Cubo, Vol. 21, No. 1, 21-35, 2019. Online: https://doi.org/10.4067/S0719-06462019000100021

[5] Y. J. Bagul, and S. K. Panchal, Certain inequalities of Kober and Lazarević type, 21(2018), Art. 137, 8 pp.

[6] G. Bercu, Sharp bounds on the sinc function via the Fourier series method, Journal of Mathematical Inequalities, Vol. 13, No. 2 pp. 495-504, 2019. Online: https://doi.org/10.7153/jmi-2019-13-33

[7] B. A. Bhayo, R. Klén and J. Sándor, New trigonometric and hyperbolic inequalities, Miskolc Math. Notes, Vol. 18, No. 1, pp. 125-137, 2017.

[8] B. A. Bhayo and J. Sándor, On certain old and new trigonometric and hyperbolic inequalities, Analysis Mathematica, Vol. 41, 2015, pp. 3-15. Doi: 10.1007/s10476-015-0102-9

[9] C. Chesneau, and Y. J. Bagul, A note on some new bounds for trigonometric functions using infinite products, Preprint Hal. 2018, (hal-01934571v2) 
[10] R. M. Dhaigude, C. Chesneau, and Y. J. Bagul, About trigonometric-polynomial bounds of sinc function, Math. Sci. Appl. E-Notes, Vol. 8, No. 1, pp. 100-104, 2020. Online: https://doi.org/10.36753/mathenot.585735

[11] V. Heikkala, M. K. Vamanamurthy, and M. Vuorinen, Generalized elliptic integrals, Comput. Methods Funct. Theory, Vol. 9, No. 1, pp. 75-109, 2009.

[12] A. Jeffrey, Handbook of Mathematical Formulas and Integrals, $3^{\text {rd }}$ edition, Elsevier Academic Press, San Diego, CA, 2004.

[13] R. Klén, M. Visuri and M. Vuorinen, On Jordan type inequalities for hyperbolic functions, J. Inequal. Appl., Vol. 2010, Article no. 362548, 2010.

[14] M. K. Kwong, On Hopital-style rules for monotonicity and oscillation, arXiv: 1502.07805[math.CA], 2015.

[15] B. Malešević, T. Lutovac, M. Rašajski, and B. Banjac, Double-sided Taylor's approximations and their applications in theory of trigonometric inequalities, in 'Trigonometric Sums and Teir Applications' ed. by A. Raigorodskii and M. T. Rassias, Springer, Cham, pp. 159-167, 2020. Online: https://doi.org/10.1007/978-3-030-37904-9

[16] E. Neuman, Refinements and generalizations of certain inequalities involving trigonometric and hyperbolic functions, Advances in Inequalities and Applications, Vol. 1, No. 1, pp. 1-11, 2012.

[17] F. Qi, A double inequality for the ratio of two non-zero neighbouring Bernoulli numbers, Journal of Computational and Applied Mathematics, Vol. 351, 1 May, pp. 1-5, 2019.

[18] F. Qi, D.-W. Niu, and B.-N. Guo, Refinements, Generalizations and Applications of Jordans inequality and related problems, Journal of Inequalities and Applications, Vol. 2009, Article ID 271923, 52 pp., 2009.

[19] J. Sándor and B. A. Bhayo, On an inequality of Redheffer, Miskolc Mathematical Notes, Vol. 16, No. 1, 2015, pp. 475-482.

[20] J. S. Sumner, A. A. Jagers, M. Vowe, and J. Anglesio, Inequalities involving trigonometric functions, The American Mathematical Monthly, Vol. 98, No. 3, 1991, pp. 264-267.

[21] L. Zhu and J. Sun, Six new Redheffer-type inequalities for circular and hyperbolic functions, Comput. Math. Appl., Volume 56, pp. 522-529, 2008.

Department of Mathematics, K. K. M. College, Manwath, Dist : Parbhani(M.S.) - 431505, INDIA

E-mail address: yjbagul@gmail.com

LMNO, University of Caen-Normandie, Caen, France

E-mail address: christophe.chesneau@unicaen.fr 